Document downloaded from:

http://hdl.handle.net/10251/35654

This paper must be cited as:

Guijarro Tarradellas, E.; Cardós, M.; Babiloni, E. (2012). On the exact calculation of the fill rate in a periodic review inventory policy under discrete demand patterns. European Journal of Operational Research. 218(2):442-447. doi:10.1016/j.ejor.2011.11.025.

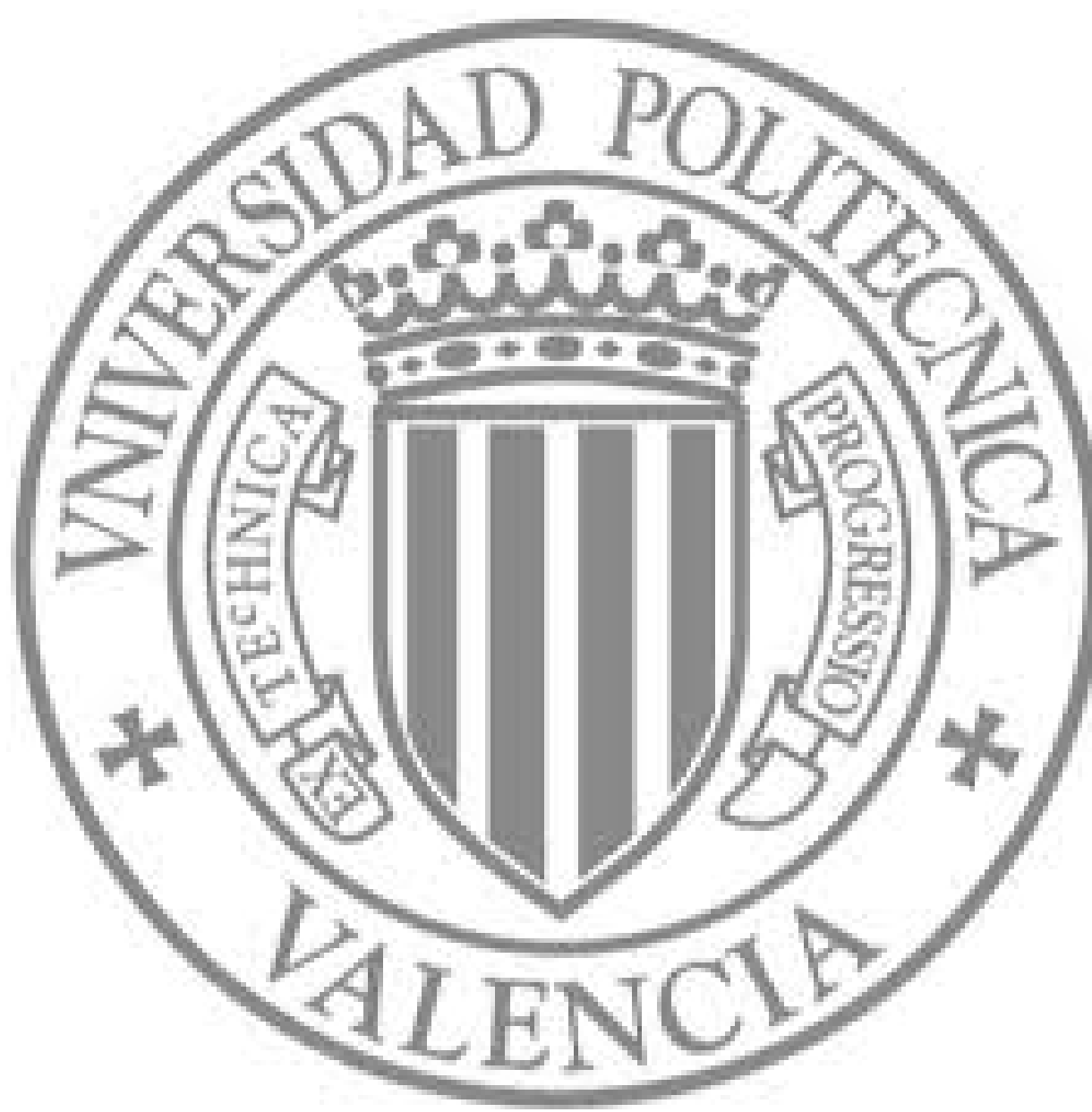

The final publication is available at

http://dx.doi.org/10.1016/j.ejor.2011.11.025

Copyright Elsevier 


\title{
On the exact calculation of the fill rate in a periodic review inventory policy under discrete demand patterns
}

\author{
GUIJARRO, E., CARDÓS, M., BABILONI, E. \\ Universidad Politécnica de Valencia
}

\begin{abstract}
The primary goal of this paper is the development of a generalized method to compute the fill rate for any discrete demand distribution in a periodic review policy. The fill rate is defined as the fraction of demand that is satisfied directly from shelf. In the majority of related work, this service metric is computed by using what is known as the traditional approximation, which calculates the fill rate as the complement of the quotient between the expected unfulfilled demand and the expected demand per replenishment cycle, instead of focusing on the expected fraction of fulfilled demand. This paper shows the systematic underestimation of the fill rate when the traditional approximation is used, and revises both the foundations of the traditional approach and the definition of fill rate itself. As a result, this paper presents the following main contributions: (i) a new exact procedure to compute the traditional approximation for any discrete demand distribution; (ii) a more suitable definition of the fill rate in order to ignore those cycles without demand; and (iii) a new standard procedure to compute the fill rate that outperforms previous approaches, especially when the probability of zero demand is substantial. This paper focuses on the traditional periodic review, order up to level system under any uncorrelated, discrete and stationary demand pattern for the lost sales scenario.
\end{abstract}

Keywords: inventory, fill rate, periodic review, discrete demand, lost sales. 


\section{Introduction and literature review}

One of the service measures most commonly used in practice is the volume fill rate, defined as the fraction of demand that is immediately fulfilled from on hand stock (see Silver et al. (1998) or Axsäter (2000) among others). Therefore, in practice it also indicates the size of the backordering demand when this is allowed (Tempelmeier (2007)). The volume fill rate is also known as item fill rate, unit fill rate or just fill rate (denoted by $\beta$ in the rest of this paper). Over the last sixty years, several works have suggested methods to estimate the fill rate in different contexts. However, to the best of our knowledge, there is not any exact and general method to estimate it under any discrete demand context. This paper focuses on the exact estimation of the fill rate for the periodic review, order up to level (base stock) system. This stock policy is commonly denoted by $(R, S)$ and consists of examining the status of an item every $R$ fixed time periods and launching a replenishment order which raises the inventory position to the order up to level $S$.

Traditionally the estimation of the fill rate is simplified through the computation of the number of units short, i.e. the demand that is not satisfied, instead of computing directly the fulfilled demand per replenishment cycle. This approach was named as the traditional approximation (denoted by $\beta_{\text {Approx }}$ in the rest of this paper) by Johnson et al. (1995) and consists of calculating the complement of the quotient between the expected unfulfilled demand per replenishment cycle and the total expected demand per replenishment cycle as follows

$$
\beta_{\text {Approx }}=1-\frac{E(\text { unfulfilled demand per replenishment cycle })}{E(\text { total demand per replenishment cycle })}
$$

Given that the expected demand per replenishment cycle can be easily calculated, methods based on the traditional approach focus on estimating the expected unfulfilled demand per replenishment cycle (also known as expected shortage). Additionally most of the authors assume a normally distributed demand for the $(R, S)$ system, e.g. Hadley and Whitin (1963), Silver and Peterson (1985), de Kok (1990), Johnson et al. (1995) and Silver and Bischak (2011). Even those authors that define the fill rate as the long run average fraction of demand satisfied immediately from shelf, estimate it by using the traditional approximation [Sobel (2004); Zhang and Zhang (2007) and Chen et al. (2003)]. In fact, Teunter (2009) demonstrates the equivalence between the long run approach and the traditional approximation. Then, the study of the related literature reveals that the traditional approximation is the most common method used to compute the fill rate. However there is not 
available a general and exact expression to calculate the fill rate when demand is modelled with any discrete demand pattern for the $(R, S)$ system.

Another approach to compute the fill rate consists of directly estimating the fraction of the fulfilled demand per replenishment cycle instead of determining the expected shortage. But in this case as well, estimation methods are developed just for specific demand distributions and inventory systems, as is the case of Feeney and Sherbrooke (1966), who develop an exact method for the sales replacement policy $(S-1, S)$ when demand follows a compound Poisson process, which is later simplified by Muckstadt and Thomas (1980) for the Poisson distribution. On the other hand, Boyaci and Gallego (2001) develop an estimation method of the fill rate under Poisson demand distribution when the inventory is continuously reviewed in an order point, order up to level $(s, S)$ system.

\begin{tabular}{c|c|c|c|c}
\hline Reference & $\begin{array}{c}\text { Inventory } \\
\text { Policy }\end{array}$ & $\begin{array}{c}\text { Demand } \\
\text { Pattern }\end{array}$ & $\begin{array}{c}\text { Fill Rate } \\
\text { Approach }\end{array}$ & Exact \\
\hline Hadley and Whitin (1963) & $(R, S)$ & Normal & TA & $\mathrm{y}$ \\
\hline Feeney and Sherbrooke (1966) & $(S-1, S)$ & Poisson & TA & $\mathrm{y}$ \\
\hline Muckstadt and Thomas (1980) & $(S-1, S)$ & Poisson & FD & $\mathrm{y}$ \\
\hline Silver and Peterson $(1985)$ & $(R, S)$ & Normal & TA & $\mathrm{n}$ \\
\hline de Kok (1990) & $(R, S)$ & Normal & TA & $\mathrm{y}$ \\
\hline Johnson et al. (1995) & $(R, S)$ & Normal & TA & $\mathrm{y}$ \\
\hline Boyaci and Gallego (2001) & $(s, S)$ & Poisson & FD & $\mathrm{y}$ \\
\hline Sobel (2004) & $(R, S)$ & Any continuous & TA & $\mathrm{y}$ \\
\hline & & Gamma & TA & $\mathrm{y}$ \\
\hline & & Normal & TA & $\mathrm{y}$ \\
\hline Zhang and Zhang $(2007)$ & $(R, S)$ & Any continuous & TA & $\mathrm{y}$ \\
\hline & & Normal & TA & $\mathrm{y}$ \\
\hline Teunter (2009) & $(R, S)$ & Any continuous & TA & $\mathrm{y}$ \\
\hline Silver and Bischak $(2011)$ & $(R, S)$ & Normal & TA & $\mathrm{y}$ \\
\hline
\end{tabular}

Approach: Traditional approximation (TA), Expected fraction of fulfilled demand (FD). Exact: yes (y), no (n)

Table 1: Overview of approaches and methods to compute the fill rate.

Table 1 summarizes the different methods and approaches previously cited that are used to compute the fill rate. This paper proposes for the $(R, S)$ system, for any discrete demand pattern: (1) a general method to compute the traditional approximation; and (2) a general method to compute the fill rate as the fraction of demand that is immediately fulfilled from 
shelf. In order to achieve these objectives, the fill rate definition is revised to avoid cycles without demand. Furthermore this paper assumes that unfulfilled demand is lost, that is the most common situation in certain industries such as the retail sector (Johansen (2005)). The remaining Sections of this paper are organized as follows. The periodic review policy, notation and assumptions of this paper are introduced in Section 2. Section 3 is dedicated to the derivation of a standard method to compute the expected unfulfilled demand per replenishment cycle and to test the performance of the traditional approximation. Section 4 focuses on the fill rate definition and derives a revised method to compute the fill rate, based on this definition. However, the lack of accuracy that the revised approach shows for patterns with a high probability of zero demand leads us to review the fill rate definition in Section 5, and to derive a generalized method able to provide the exact value of the fill rate even when the probability of no demand during the cycle cannot be ignored. Finally, Section 6 is dedicated to the discussion of the methods presented in Sections 3, 4 and 5 and to summarize the main contributions of this paper.

\section{Basic notation and assumptions}

The traditional periodic review, order up to level $(R, S)$ system places replenishment orders every $R$ units of time of sufficient magnitude to raise the inventory position to the order up to level $S$. The replenishment order is received $L$ periods after being launched. Figure 1 shows an example of the evolution of the on hand stock in this system. Notation in the rest of the paper is as follows

$S \quad=\quad$ order up to level (units),

$R=$ review period corresponding to the time between two consecutive reviews and replenishment cycle corresponding to the time between two consecutive deliveries (time units),

$L=$ lead time for the replenishment order,

$z_{t}=$ on hand stock at time $t$ from the first reception,

$D_{t} \quad=\quad$ total demand during $t$ consecutive periods,

$f_{t}(\cdot)=$ probability mass function of $D_{t}$,

$F_{t}(\cdot)=$ cumulative distribution function of $D_{t}$, 


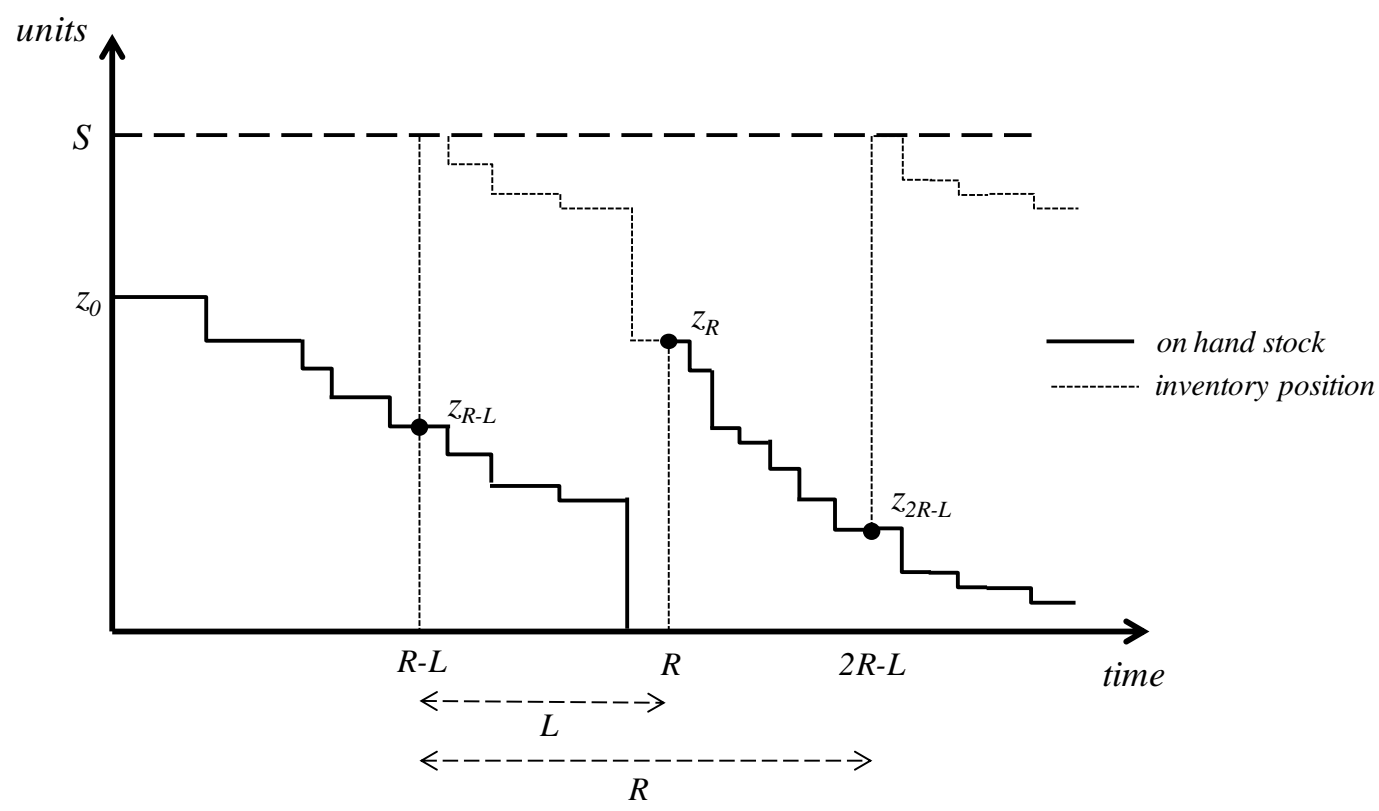

Figure 1: On hand stock and inventory position evolution in a periodic review, lost sales order up to level system

The rest of the paper assumes that: (i) time is discrete and is organized in a numerable and infinite succession of equi spaced instants; (ii) the lead time, $L$, is constant; (iii) only one outstanding replenishment order is launched within any period which means that $L<R$; (iv) backlogged demand is not allowed; (v) the replenishment order is added to the inventory at the end of the period in which it is received, hence these products are available for the next period; (vi) demand during a period is fulfilled with the on hand stock at the beginning of the period; and (vii) the demand process is considered stationary, independent and identically distributed, i.i.d., and defined by any discrete function. Note that assumption (iii) is widely used in the common derivation of policies for the lost sales scenario. The complexity to extend it to more than one replenishment order is explained in Hadley and Whitin (1963).

\section{Standard method to compute the traditional approximation}

The traditional approximation of the fill rate computes the complement of the ratio between the expected unfulfilled demand and the expected demand per replenishment cycle as shown in expression (1). The expected demand can be straightforwardly computed so all that is left to compute is the expected unfulfilled demand per replenishment cycle. However, as the introduction points out, the related literature only suggests expressions to compute it for specific demand distributions. Section 3.1 is dedicated to the proposal of a standard method for the calculation of the expected unfulfilled demand that can be used when demand follows any discrete demand distribution, according to the assumptions detailed in the previous 
section. After that, Section 3.2 shows the performance of the method comparing with simulated data.

\subsection{Derivation}

The unfulfilled demand per replenishment cycle depends on both the amount of demand during the cycle, $D_{R}$, and the on hand stock level just after the replenishment arrives at the beginning of the cycle, $z_{0}$. Obviously, under periodic review conditions, a shortage occurs whenever $D_{R}>z_{0}$ and hence, for any feasible value of $z_{0} \in[0, S]$, the expected unfulfilled demand per cycle is as follows

$E($ unfulfilled demand per replenishment cycle $)=\sum_{i=0}^{S} P\left(z_{0}=i\right) \cdot \sum_{j=i+1}^{\infty}(j-i) \cdot P\left(D_{R}=j\right)$

The complexity of (2) is, in fact, in how to compute the probability of every stock level at the beginning of the cycle i.e., the whole vector $\overline{P\left(z_{0}\right)}$. Note that we assume that the on hand stock at the beginning of the cycle can be from 0 to $S$. To estimate the probability of every $z_{0}$, we follow the inductive approach proposed by Cardós et al. (2006) which is further explained in Cardos and Babiloni (2011). This probability vector is computed by means of calculating the probability transition matrixes of the on hand stock levels between: (i) the beginning of the cycle and the review (times $O$ and $R-L$ in Figure 1); and (ii) the review and the beginning of the next replenishment cycle (times $R-L$ and $R$ in Figure 1). In summary, the computational effort to find the pmf of $z_{0}$ is substantial. Once $\overline{P\left(z_{0}\right)}$ is known, substituting expression (2) into expression (1), the standard method to compute the traditional approximation of the fill rate can be expressed as $\beta_{\text {Approx }}=1-\frac{\sum_{i=0}^{S} P\left(z_{0}=i\right) \cdot \sum_{j=i+1}^{\infty}(j-i) \cdot f_{R}(j)}{\sum_{j=1}^{\infty} j \cdot f_{R}(j)}$

where the denominator represents the expected total demand per replenishment cycle.

\subsection{Illustrative examples and discussion}

This section illustrates the performance of expression (3) against the simulated fill rate, $\beta_{\text {Sim }}$, which is computed as the average fraction of the fulfilled demand in every replenishment cycle when considering 20,000 consecutive periods:

$$
\beta_{\text {Sim }}=\frac{1}{T} \sum_{t=1}^{T} \frac{\text { fulfilled demand }_{t}}{\text { total demand }_{t}}
$$


where $T$ indicates the total number of replenishment cycles. This simulation uses the data from Table 2 which encompasses 54 different cases.

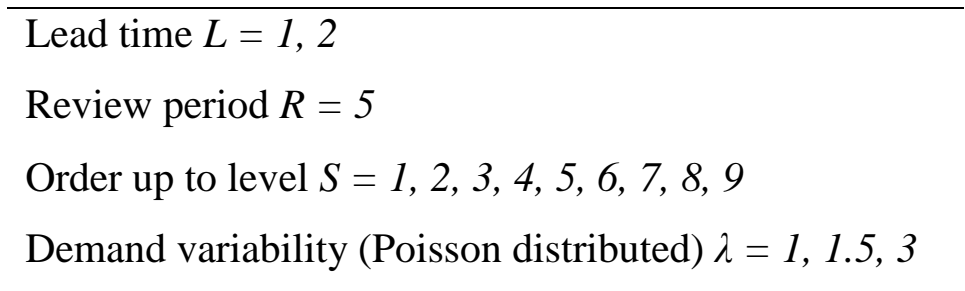

Table 2: First set of data (54 cases)

Figure 2 presents the comparison between $\beta_{\text {Approx }}$ and $\beta_{\text {Sim }}$ for the Table 2 cases and shows the significant deviation that appears between them. In fact, $\beta_{\text {Approx }}$ underestimates the simulated fill rate for all the cases and therefore the traditional approximation seems to be biased. When demand is normally distributed, Johnson et al. (1995) pointed out results similar to those we obtained for the Poisson distribution. Hence, the traditional approximation produces significant deviations that cannot be neglected. Note that the expression (3) leads to the exact value of the traditional approximation. Therefore deviations that Figure 2 shows arise from estimating the fill rate using the traditional approach (see expression (1)) and not from how it is calculated.

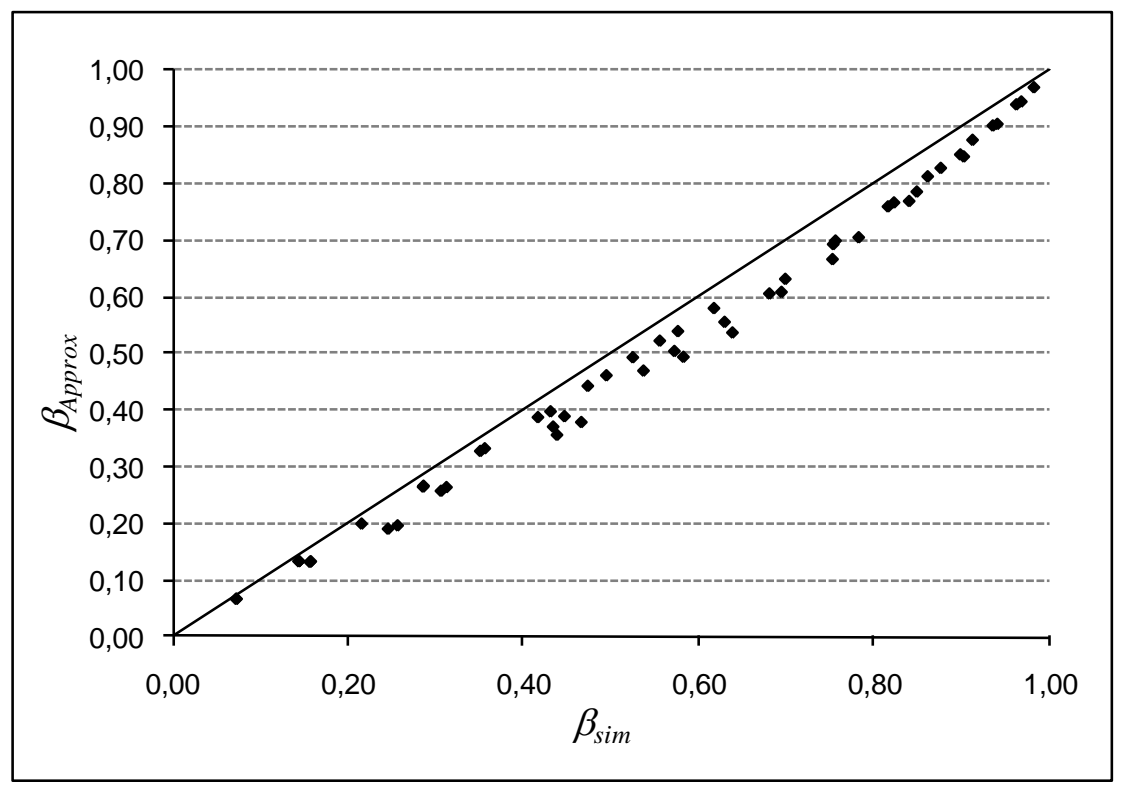

Figure 2: $\beta_{\text {Approx }}$ vs. $\beta_{\text {Sim }}$ for the cases from Table 2

\section{A revised approach focused on the fill rate definition}

In an effort to understand the lack of accuracy the traditional approximation shows, we realize that expression (1) does not really fit the fill rate definition. As mentioned, the fill rate is 
defined as the fraction of the demand that is fulfilled immediately from the on hand stock. Therefore, according to the definition, the fill rate is

$$
E\left(\frac{\text { fulfilled demand per replenishment cycle }}{\text { total demand per replenishment cycle }}\right)
$$

As known, $E(X \cdot Y)=E(X) \cdot E(Y)$ if $X$ and $Y$ are independent random variables. Even in this context, $E\left(\frac{X}{Y}\right)=E(X) \cdot E\left(\frac{1}{Y}\right)$ but $E\left(\frac{1}{Y}\right) \neq \frac{1}{E(Y)}$ and therefore $E\left(\frac{X}{Y}\right) \neq \frac{E(X)}{E(Y)}$ (see for example Grinstead and Snell (1997)).

Therefore,

$E\left(\frac{\text { fulfilled demad per replenishment cycle }}{\text { total demand per replenishment cycle }}\right) \neq \frac{E(\text { fulfilled demad per replenishment cycle })}{E(\text { total demand per replenishment cycle })}$

note that

$\frac{E(\text { fulfilled demand per replenishment cycle })}{E(\text { total demand per replenishment cycle })}=1-\frac{E(\text { unfulfilled demand per replenishment cycle })}{E(\text { total demand per replenishment cycle })}$

and then

$E\left(\frac{\text { fulfilled demand per replenishment cycle }}{\text { total demand per replenishment cycle }}\right) \neq 1-\frac{E(\text { unfulfilled demand per replenishment cycle })}{E(\text { total demand per replenishment cycle })}$

This section is dedicated to derive an exact method to estimate the fill rate following the expression (5) for the $(R, S)$ system valid for any discrete distribution of demand. This method is named as $\beta_{R e v}$ and its performance over different Poisson demand patterns is analyzed through some illustrative examples in Section 4.2.

\subsection{Derivation}

Demand during a replenishment cycle can be: (i) lower than or equal to the on hand stock at the beginning of the cycle, i.e. $D_{R} \leq z_{0}$. Then the fill rate will be equal to 1 ; or (ii) greater than the on hand stock at the beginning of this cycle, i.e. $D_{R}>z_{0}$. Then the fill rate will be the fraction of this demand that is satisfied by the on hand stock at the beginning of this cycle. Therefore, if we know exactly the on hand stock level at the beginning of the cycle, $z_{0}=i$, then $\beta_{\text {Rev }}$ can be calculated as 


$$
\beta_{\operatorname{Rev}}\left(z_{0}=i\right)=P\left(D_{R} \leq i\right)+\sum_{j=i+1}^{\infty} \frac{i}{j} \cdot P\left(D_{R}=j\right)
$$

Expressing (6) through the probability mass and cumulative distribution functions, $f_{t}(\cdot)$ and $F_{t}(\cdot)$ respectively,

$$
\beta_{\mathrm{Rev}}\left(z_{0}=i\right)=F_{R}(i)+\sum_{j=i+1}^{\infty} \frac{i}{j} \cdot f_{R}(j)
$$

Finally, applying expression (7) to every stock level at the beginning of the cycle $z_{0} \in[0, S]$ the general method to compute $\beta_{R e v}$ is

$$
\beta_{\mathrm{Re} v}=\sum_{i=0}^{S} P\left(z_{0}=i\right) \cdot\left(F_{R}(i)+\sum_{j=i+1}^{\infty} \frac{i}{j} \cdot f_{R}(j)\right)
$$

\subsection{Illustrative examples and discussion}

Using the data from Table 2, Figure 3 shows the comparison between $\beta_{R e v}$ and $\beta_{S i m}$ where neither bias nor significant deviations appear. Therefore, the suggested revised method outperforms the traditional approximation for these cases.

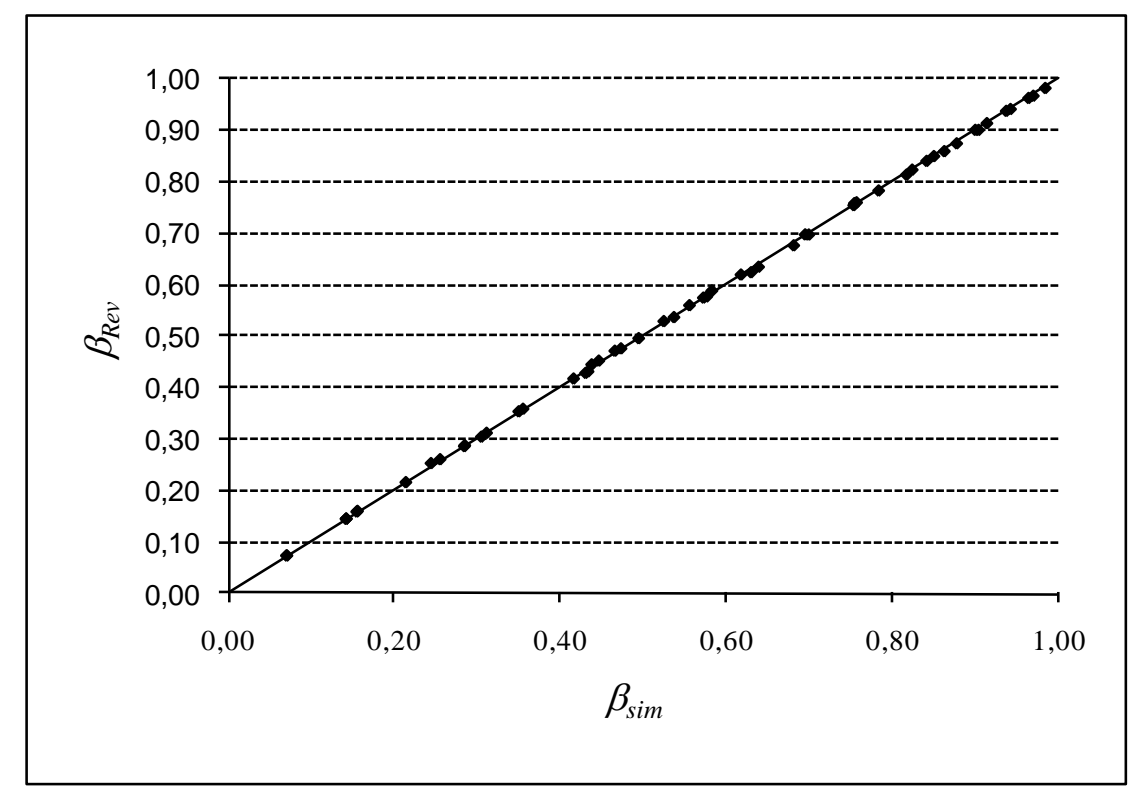

Figure 3: $\beta_{\text {Rev }}$ vs. $\beta_{\text {Sim }}$ for the cases from Table 2

However, with the aim of testing the performance of $\beta_{R e v}$ over different demand patterns we use a new set of data that includes Poisson distributions with a high probability of zero 
demand over the replenishment cycle. Figure 4 illustrates the performance of $\beta_{\operatorname{Sim}}$ versus $\beta_{\text {Rev }}$ for the 54 cases which result from the set of data summarized in Table 3.

Lead time $L=1,2$

Review period $R=5$

Order up to level $S=1,2,3,4,5,6,7,8,9$

Demand rate (Poisson distributed) $\lambda=0.01,0.1,0.5$

Table 3: Second set of data (54 cases)

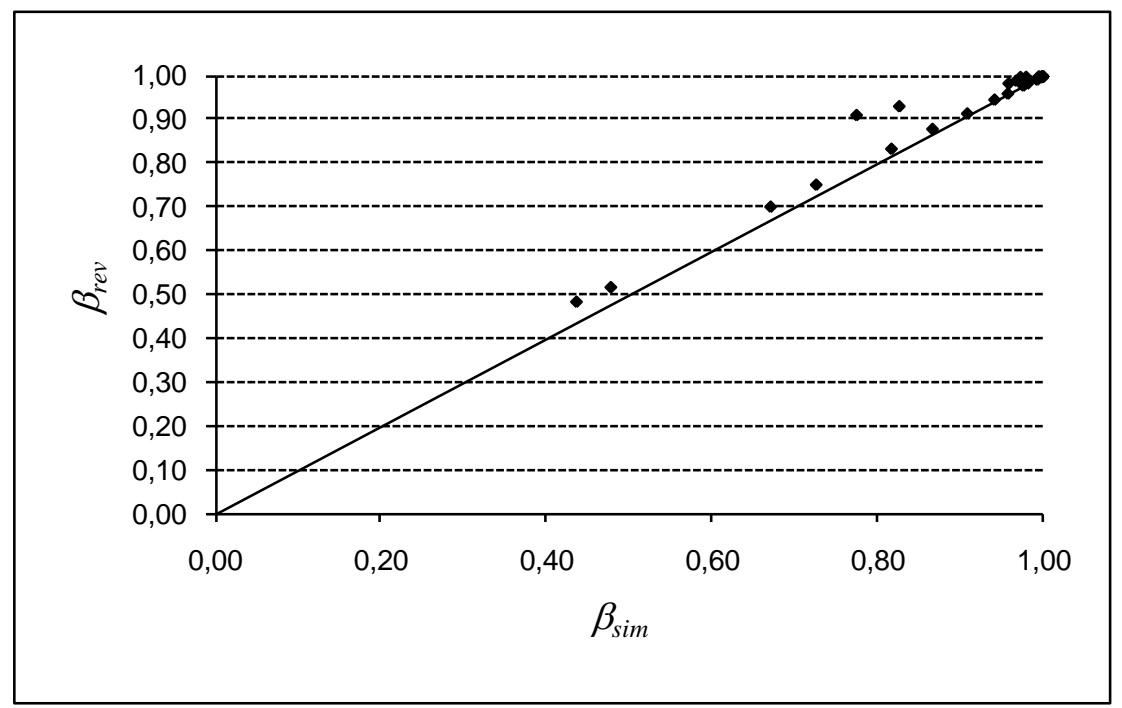

Figure 4: $\beta_{\text {Rev }}$ vs. $\beta_{\text {Sim }}$ for the cases from Table 3

Figure 4 shows the following main results: (i) significant deviations appear between $\beta_{R e v}$ and $\beta_{\text {Sim }}$; and (ii) $\beta_{\text {Rev }}$ tends to overestimate $\beta_{\text {Sim }}$. We find that during the cycles without demand expression (8) leads to consider the fill rate equal to 1 . As a consequence of this reasoning, $\beta_{\text {Rev }}$ always overestimates $\beta_{\text {Sim }}$ in those cycles. However, from a practical point of view, it is useless to consider a service metric when there is no demand to be served. For example, consider a sku whose stock is reviewed monthly over a year and with no demand during 11 months. The fill rate per cycle will be equal to 1 if it is estimated through expression (8). Suppose now that two units are demanded in month 12 but there is only one available unit on the shelf. The fill rate for this last month will be 0.50 . After that, the annual fill rate for this sku will be equal to 0.96 when in fact just half of the demanded units per year have been served. Therefore, computing the fill rate using expression (8) does not show the real performance of the inventory system. As a consequence cycles without demand should not be 
taken into account to estimate the fill rate. Thus, although $\beta_{R e v}$ is more accurate than the traditional approximation, it cannot be accepted as a general method to estimate the fill rate.

\section{The proposed approach: a generalized method to estimate the fill rate}

In order to overcome the shortcoming explained above, we need to include explicitly the condition of having positive demand during the cycle in the fill rate definition. Therefore, we propose a generalized definition of the fill rate as the fraction of demand that is fulfilled with the on hand stock during a cycle with positive demand, which is expressed as

$$
E\left(\frac{\text { fulfilled demand per replenishment cycle }}{\text { total demand per replenishment cycle }} \mid \text { positive demand during the cycle }\right)
$$

Sections 5.1 and 5.2 are dedicated to the derivation of a generalized method named as $\beta^{*}$ to compute the fill rate according to the expression (9), and to measure its performance, respectively.

\subsection{Derivation}

Following the same reasoning as in Section 4.1., if we know the on hand stock level $z_{0}$ at the beginning of the cycle, we can calculate its fill rate $\beta^{*}$ using the following expression

$$
\beta^{*}\left(z_{0}=i \mid D_{R}>0\right)=P\left(D_{R} \leq i \mid D_{R}>0\right)+\sum_{j=i+1}^{\infty} \frac{i}{j} \cdot P\left(D_{R}=j \mid D_{R}>0\right)
$$

Note that this expression is the same as the expression (6), but including the condition of positive demand during the replenishment cycle. Using the probability mass function and the cumulative distribution function, we can rewrite (10) as follows

$$
\beta^{*}\left(z_{0}=i \mid D_{R}>0\right)=\frac{F_{R}(i)-F_{R}(0)}{1-F_{R}(0)}+\sum_{j=i+1}^{\infty} \frac{i}{j} \cdot \frac{f_{R}(j)}{1-F_{R}(0)}
$$

Extending expression (11) to every feasible value of $z_{0}$, the generalized expression to compute $\beta^{*}$ results as

$$
\beta^{*}=\sum_{i=0}^{S} P\left(z_{0}=i\right) \cdot\left\{\frac{F_{R}(i)-F_{R}(0)}{1-F_{R}(0)}+\sum_{j=i+1}^{\infty} \frac{i}{j} \cdot \frac{f_{R}(j)}{1-F_{R}(0)}\right\}
$$




\subsection{Illustrative examples and discussion}

Figure 5 shows the comparison between $\beta^{*}$ and $\beta_{\text {Sim }}$ for the resultant cases from Table 2 and Table 3. As can be observed neither bias nor significant deviations appears on it for any of the 108 aggregated cases. Therefore in these cases $\beta^{*}$ seems to compute accurately the fill rate even when the probability of no demand during the cycle cannot be neglected.

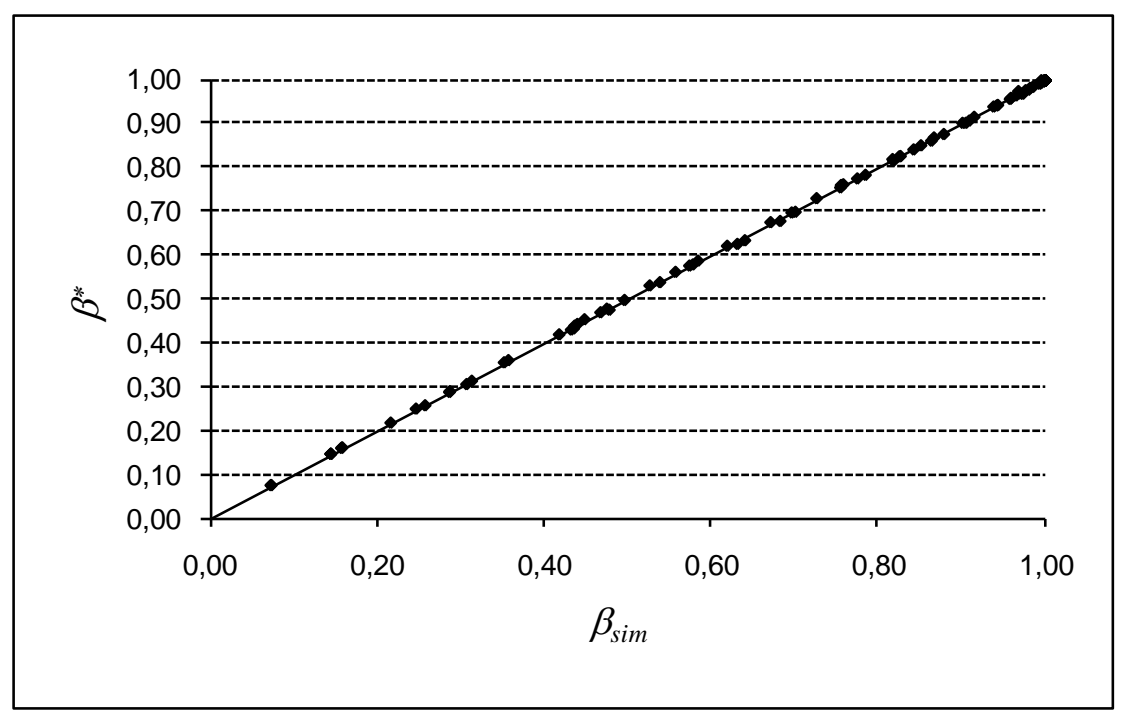

Figure 5: $\beta^{*}$ vs. $\beta_{\text {Sim }}$ for the cases from Table 2 and Table 3

\section{Discussion and summary}

The traditional approximation of the fill rate, $\beta_{\text {Approx }}$, computes it by estimating the ratio between the expected unfulfilled demand per replenishment cycle and the total expected demand per replenishment cycle. According to the related literature, the difficulty consists of obtaining the expected unfulfilled demand (expected shortage) during the replenishment cycle. We presented in Section 3 a standard method to calculate the exact expected unfulfilled demand per replenishment cycle and use it to compute $\beta_{\text {Approx }}$ for any discrete demand distribution. However, when we tested its performance versus the simulated fill rate (see Figure 2), we realised that the traditional approximation tends to underestimate the simulated fill rate, and therefore the traditional approximation is biased. An important consequence of this is found when using a target fill rate to determine the order up to level of the inventory policy. Figure 6 shows the evolution of the traditional approximation, $\beta_{\text {Approx }}$, and the exact estimation, $\beta^{*}$, when increasing the order up to level for a Poisson distributed demand with demand rate $\lambda=1$, review period $R=5$ and lead time $L=2$. It can be seen that if, for example, a target fill rate is set to 0.70 , the traditional approximation leads to $S=5$ whereas in fact just $S=4$ is necessary to reach the target. In this example, using the traditional approximation to 
determine $S$ leads to an increase in the average stock level and thus the inventory holding cost of the system. This inefficiency is especially relevant in industries in which the unit cost of the item is high and/or storage space is limited. Therefore, managers should be aware of the risk of using the traditional approximation to set the order up to level.

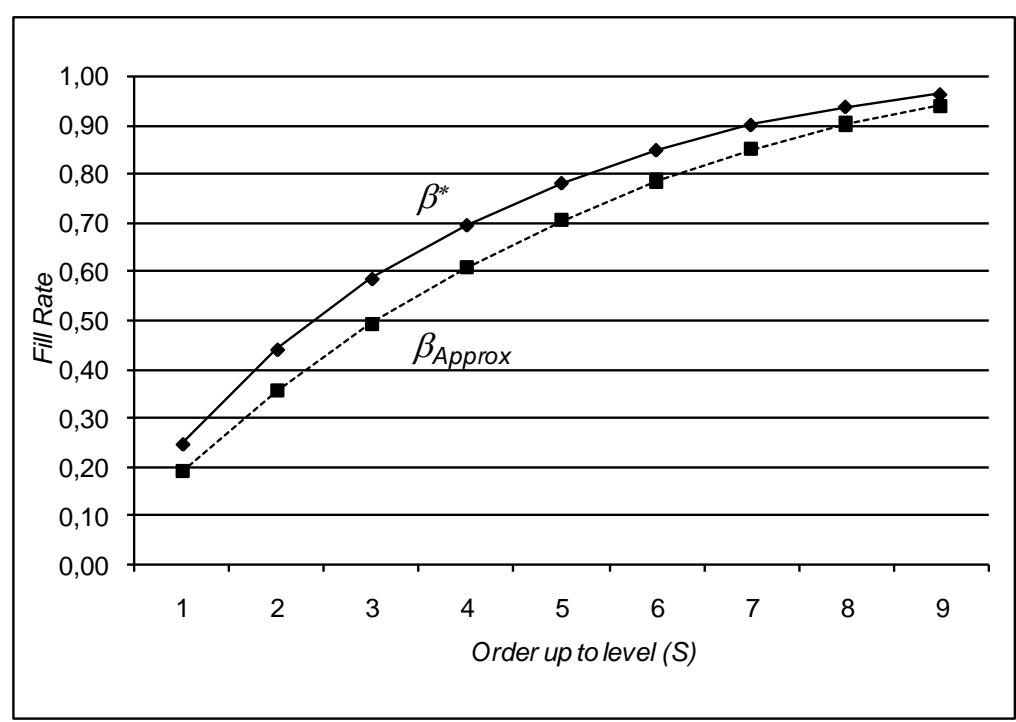

Figure 6: Comparison between the traditional approximation, $\beta_{\text {Approx }}$, and the exact estimation, $\beta^{*}$, with Poisson demand with $\lambda=1, R=5$ and $L=2$.

In order to improve the fill rate estimation we later focused on the fill rate definition and suggest a revised method, $\beta_{R e v}$, to directly estimate the expected fulfilled demand per replenishment cycle exactly as stated by the definition itself. This method works properly in some cases, as Figure 3 shows; however the simulated fill rate is overestimated by $\beta_{R e v}$ when dealing with demand patterns with a significant probability of zero demand, as in Figure 4. These results lead us to go back again to the fill rate definition which is generalized in Section 5 to explicitly avoid the chance of no demand during the cycle. In keeping with this, a new generalized and exact estimation method, $\beta^{*}$, is proposed in Section 5 to compute the fill rate with any discrete demand pattern, as illustrated by Figure 5. Therefore, the generalized fill rate method proposed in this paper, $\beta^{*}$ : (i) leads to the exact fill rate value; (ii) is suitable even when the probability of zero demand cannot be neglected; (iii) can be applied to any discrete demand distribution. Note that the need to consider only cycles with positive demand does not emerge when using the traditional approximation because it just considers the expected demand, and in the case of no demand cycles it does not affect the estimation. This paper proposes a revised definition of the fill rate in a discrete demand context to avoid the distortion caused by the cycles with no demand. However, the revised definition also 
applies when demand is continuous because the cycles without demand have also to be removed to obtain a useful and general service metric. In fact, the exact estimation procedures that can be found in the literature for both discrete or continuous demand can only be applied when the chance of no demand during the cycle is negligible as pointed out by Silver and Bischak (2011).

This paper is part of a wider research project devoted to identify the most simple and effective method to find the lowest value of $S$ which ensures the target fill rate under discrete context. Therefore, further extensions should be focused on: (i) assessing the exact method when using other discrete distribution functions of demand able to model significant variability on demand sizes with a high probability of zero demand; (ii) characterizing the cases where approximations (including some possible new ones) have the most important deviations, (iii) analyzing risks of using different fill rate approximations to set the parameters of the stock policy, and (iv) deriving a new expression for the backordering scenario that follows the approach suggested in this paper.

\section{Acknowledgements}

The authors would like to thank the two anonymous referees for their thorough reading and very constructive comments on earlier versions of this paper.

\section{References}

Axsäter, S. (2000). Inventory Control. (1st ed.). Norwell, Massachusetts: Kluwer Academic Publishers.

Boyaci, T., \& Gallego, G. (2001). Serial Production/Distribution Systems Under Service Constraints. Manufacturing Service Operations Management, 3, 43-50.

Cardos, M., \& Babiloni, E. (2011). Exact and approximate calculation of the cycle service level in periodic review inventory policies. International Journal of Production Economics, 131, 63-68.

Cardós, M., Miralles, C., \& Ros, L. (2006). An exact calculation of the cycle service level in a generalized periodic review system. Journal of the Operational Research Society, 57, 12521255 .

Chen, J. H., Lin, D. K. J., \& Thomas, D. J. (2003). On the single item fill rate for a finite horizon. Operations Research Letters, 31, 119-123.

de Kok, A. G. (1990). Hierarchical production planning for consumer goods. European Journal of Operational Research, 45, 55-69. 
Feeney, G. J., \& Sherbrooke, C. C. (1966). The (S-1,S) inventory policy under compound Poisson demand. Management Science, 12, 391-411.

Grinstead, C. M., \& Snell, J. L. (1997). Introduction to probability. (2nd). United States of American: AMS Bookstore.

Hadley, G., \& Whitin, T. (1963). Analysis of Inventory Systems. (Englewood Cliffs, NJ: Prentice-Hall.

Johansen, S. G. (2005). Base-stock policies for the lost sales inventory system with Poisson demand and Erlangian lead times. International Journal of Production Economics, 93-4, 429437.

Johnson, M. E., Lee, H. L., Davis, T., \& Hall, R. (1995). Expressions for Item Fill Rates in Periodic Inventory Systems. Naval Research Logistics, 42, 57-80.

Muckstadt, J. A., \& Thomas, L. J. (1980). Are Multi-Echelon Inventory Methods Worth Implementing in Systems with Low-Demand-Rate Items. Management Science, 26, 483-494.

Silver, E. A., \& Peterson, R. (1985). Decisions system for inventory management and production planning. (2nd). New York: John Wiley \& Sons.

Silver, E. A., Pyke, D. F., \& Peterson, R. (1998). Inventory Management and Production Planning and Scheduling. (3rd ed.). New York: Wiley.

Silver, E. A., \& Bischak, D. P. (2011). The exact fill rate in a periodic review base stock system under normally distributed demand. Omega International Journal of Management Science, 39, 346-349.

Sobel, M. J. (2004). Fill rates of single-stage and multistage supply systems. Manufacturing and Service Operations Management, 6, 41-52.

Tempelmeier, H. (2007). On the stochastic uncapacitated dynamic single-item lotsizing problem with service level constraints. European Journal of Operational Research, 181, 184194.

Teunter, R. H. (2009). Note on the fill rate of single-stage general periodic review inventory systems. Operations Research Letters, 37, 67-68.

Zhang, J., \& Zhang, J. (2007). Fill rate of single-stage general periodic review inventory systems. Operations Research Letters, 35, 503-509. 